\title{
Utility of a custom screw insertion guide and a full-scale, color-coded 3D plaster model for guiding safe surgical exposure and screw insertion during spine revision surgery
}

\author{
Bungo Otsuki, MD, PhD, Mitsuru Takemoto, MD, PhD, Shunsuke Fujibayashi, MD, PhD, \\ Hiroaki Kimura, MD, PhD, Kazutaka Masamoto, MD, and Shuichi Matsuda, MD, PhD \\ Department of Orthopaedic Surgery, Graduate School of Medicine, Kyoto University, Kyoto, Japan
}

Several articles have described the use of screw insertion guides during primary spine surgery; however, the use of such a guide during revision surgeries has not been described. The purpose of this study is to describe the utility of a custom screw insertion (CSI) guide assembled using a novel method and a full-scale, color-coded 3D plaster (FCTP) model for safe and accurate revision surgery.

The authors applied the CSI guide and the FCTP model in 3 cases. In the first case, a patient with multiple failed cervical spine surgeries underwent occipitocervicothoracic fusion. After a successful result for this patient, the authors applied the CSI guide in 2 other patients who underwent revision lumbar fusion surgeries to confirm the accuracy and the efficacy of the CSI guides in such cases. The models and guides were fabricated using rapid prototyping technology. The effectiveness of these methods was examined.

The FCTP model was designed using CT data. During model assembly, implants inserted during previous surgery were removed virtually, and for the cervical spine, vertebral arteries were colored red for planning. The CSI guide was designed with 5 or 6 arms to fit the bone surface precisely after removing artifacts. Surgery was performed by referring to the FCTP model. Because the actual structure of the bone surface was almost identical to that of the FCTP model, surgical exposure around the complex bone shape proceeded smoothly. The CSI guides were positioned accurately to aid the successful insertion of a pedicle screw into the C-2 vertebra in the case of cervical revision surgery, and 4 pedicle screws for lumbar vertebrae in the 2 other patients. Postoperative CT scans showed that all screw positions closely matched those predicted during the preoperative planning. In conclusion, the FCTP models and the novel CSI guides were effective for safe and accurate revision surgery of the spine.

http://thejns.org/doi/abs/10.3171/2015.12.SPINE15678

KEY WORDS pedicle screw; plaster model; screw insertion guide; rapid prototyping; revision surgery; technique

$\mathrm{W}$ ITH the development of rapid prototyping technology, several papers have described the utility of a screw insertion guide during primary spine surgery. ${ }^{3,6,7,13}$ However, no report has described the use of such a guide during revision surgery. The reported guides used in primary surgery have a large contact area designed by making a conventional subtraction cast model of the shape of the lamina and/or the spinous process from the initial bulk structure (Fig. 1). However, in revision surgery, many artifacts caused by previous internal implants, when viewed using preoperative CT, can make it difficult to design an accurate screw guide using a conventional image subtraction method. In this study, we developed a novel customized screw insertion (CSI) guide for use during revision surgery.

\section{Methods}

Segmentation Procedure for a Full-Scale, Color-Coded, 3D Plaster (FCTP) Model

All patients underwent $\mathrm{CT}$ evaluations before surgery. A contrast-enhanced CT study was also performed in a 


\section{Conventional method}
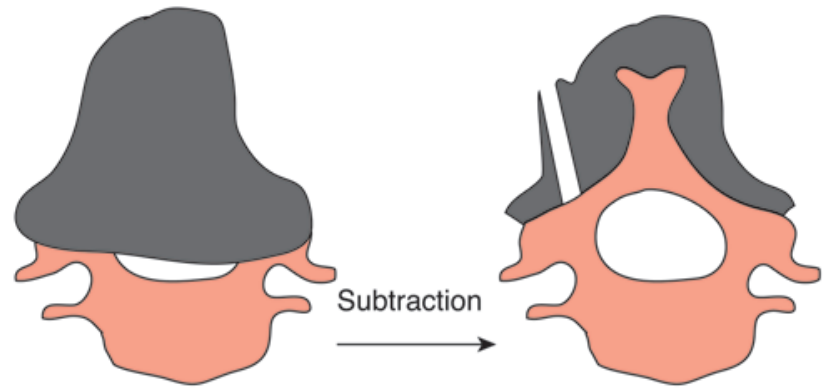

One wide contact point

\section{Our novel method}
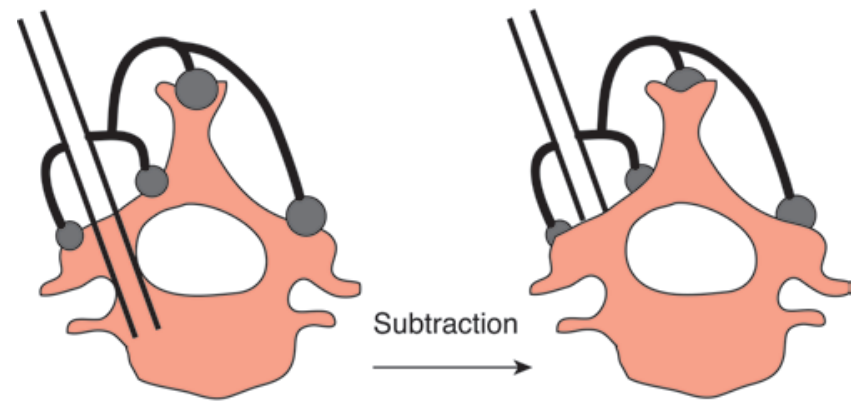

Several small contact points

FIG. 1. A schematic comparison of screw insertion guide design for the conventional method and our novel method. In the former, the screw insertion guide is designed by subtracting the bone shape from the initial bulk structure. In the latter, the guide was designed to have several appropriate small contact points that precisely fit the bone surface. Figure is available in color online only.

case involving the cervical spine (see below). The CT data (1-mm slice thickness, 0.21 - to $0.27-\mathrm{mm}$ in-plane resolution) were transferred to image-processing software (VG studio MAX 2.2; Volume Graphics). This software facilitates segmentation of the image data and 3D volume reconstruction. At first, segmentation of the internal metal implants was performed. Because metal implants have high CT contrast values distinct from that of the bone surface, they were segmented easily (Fig. 2A). Next, segmentation of the arteries (in the cervical spine case) was performed in the same way using contrast-enhanced CT data (Fig. 2B). Finally, segmentation of the bone tissue was performed. We decided the optimal threshold value for the bone surface by visual inspection (Fig. 2C). Unlike cases of primary surgery, there are artifacts caused by internal metal implants in revision cases. Usually, these artifacts are segmented with a small island-like shape (Fig. $2 \mathrm{C}$ ), and most of them can be removed virtually using erosion and dilation manipulation of the segmented area (Fig. 2C). ${ }^{11}$ The residual artifacts could be removed manually on CT images, especially around the areas where the arms of the guide would be located. These procedures are outlined in Fig. 2C. The segmented data (bone tissue, implant, and contrast medium) were converted into stereolithography format files and transferred to 3D computer-assisted design software (FreeForm, SensAble Technologies).

The protocol for using this CSI guide was approved by our institutional ethics review committee, and written informed consent was obtained from the patients after the potential risks and the aim of the study had been explained fully.

\section{Fabrication of the FCTP Models and CSI Guides}

After segmentation of the bone surface using VG Studio Max software, we identified the axial view of each intended pedicle plane, and each pedicle screw trajectory was defined as a cylindrical segment. This trajectory was confirmed using sagittal, coronal, and axial views (Fig. $3 \mathrm{~A}$ ). This maneuver is almost the same as that used in commercially available surgical navigation systems. Next, we chose the 5 or 6 appropriate bone surface areas where the arms of the guide should be located. The criteria to select each area were as follows. 1) The selected area should be located on the same continuous bone surface that connects to the screw trajectory. 2) The selected area should have high CT contrast. 3) There should be no artifacts appearing to be bony tissue around the selected area. 4) Selected areas should not be located in the same plane. 5) At least one area should be selected that can be recognized easily during surgery, such as the base of a transverse process.

After choosing the contact areas for guide arms, spherical segments with a diameter of $6-8 \mathrm{~mm}$ were located on the bone surface (Fig. 3B). The contact points of the arms were designed by subtracting the bone tissue segments from the spherical segments (Fig. 3C). These segmented data were transferred to FreeForm software as mentioned above (Fig. 4A). Using this software, pedicle screw guide tubes were designed with an outer diameter of $6 \mathrm{~mm}$ and inner diameter of $3.5 \mathrm{~mm}$ (Fig. 4B). The length of the guides was set to $30 \mathrm{~mm}$ as in our previous study. ${ }^{15}$ The contact points and the guide tube were connected with curved cylindrical supports with a diameter of $5 \mathrm{~mm}$. To prevent deformation in the 3D fabrication, these supports were reinforced by diagonal cylindrical supports (Fig. 4A and B). Finally, the stereolithography format files of the CSI guide were transferred to a selective laser-melting machine (EOSINT M270, Electro Optical Systems GmbH), and the CSI guides were fabricated using commercially available pure titanium powder (Fig. 4C). The nominal dimensional accuracy of this additive manufacturing machine is $<200 \mu \mathrm{m}$.

For FCTP fabrication, cylindrical tubes with a diameter of $3.5 \mathrm{~mm}$ corresponding to the pedicle screw trajectory were subtracted from the bone tissue, and then FCTP models were made using a gypsum-based 3D printer (ZPrinter 450, Z Corp.) (Fig. 4D). The precisions of fit and indicated insertion direction of the guide were confirmed by placement on the plaster model (Fig. 4E and F). The CSI guide was sterilized in an autoclave before being used during surgery.

\section{Surgical Procedure}

In all 3 cases, the bone surface was exposed carefully by referring to the FCTP model. The contact points on the bone surface for the guide arms were prepared. We searched for the proper guide position on the bone surface. Because there was at least one contact point that could be 

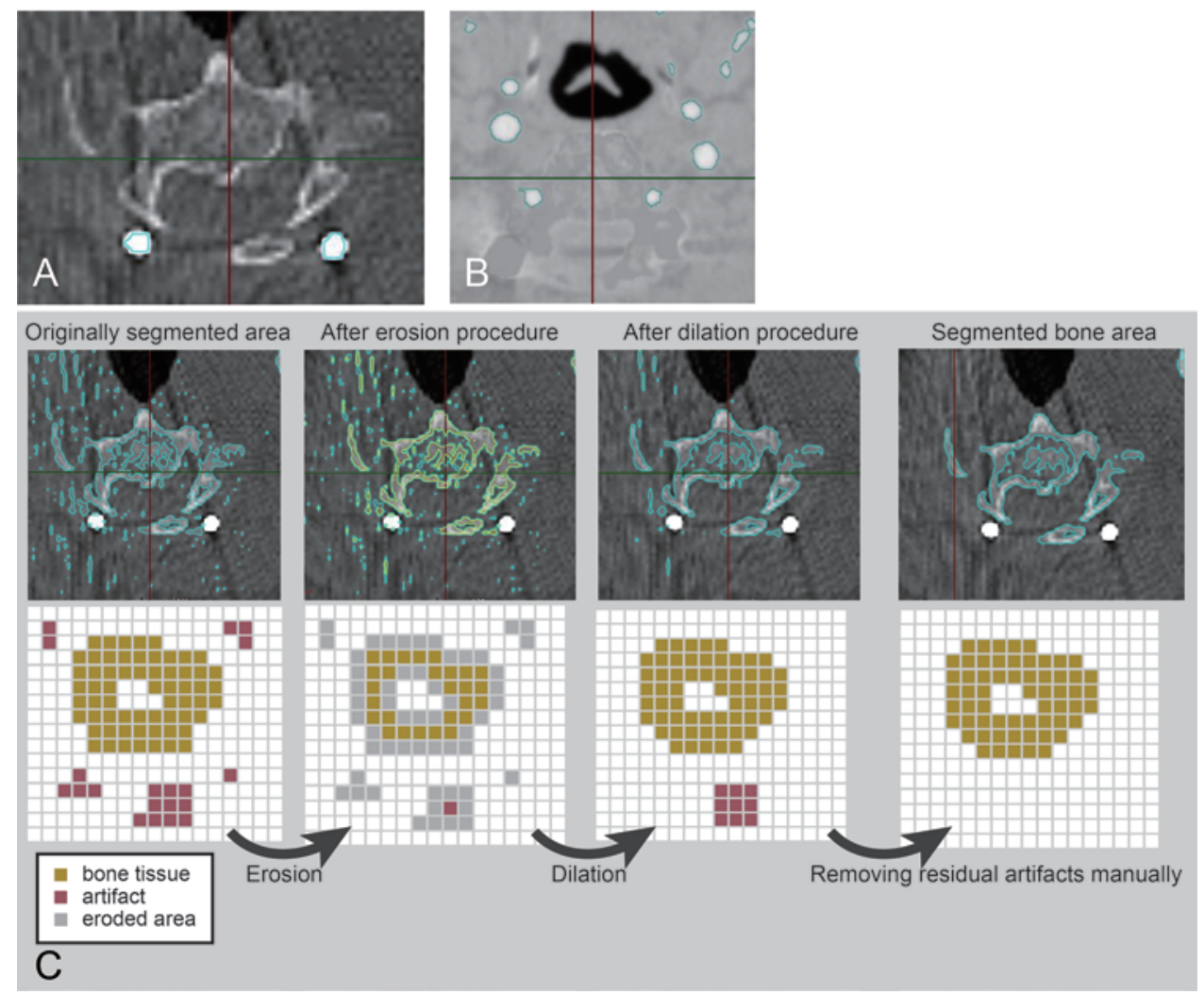

FIG. 2. Segmentation procedures. A and B: Metal implants (A) and contrast-enhanced arteries (B) are segmented according to their CT values. C: Artifacts around the bone area can be almost removed using the erosion-dilation procedure. This erosiondilation procedure can remove segments with a small area as indicated by the schematic. The remaining artifacts are removed manually one by one especially around the bone surface where the arms of the guide will be located. Figure is available in color online only.

recognized easily during surgery, the proper position of the guide could be found without any problems. Once the guides were located in their correct positions, all contact arms made contact with the bone surface, and, provided that the guides were pressed toward the bone surface manually, the guides were set without any instability. After that, in Case 1 involving the cervical spine, a pilot hole was made using a specially designed cylindrical chisel and straight pedicle probe with a diameter of $3 \mathrm{~mm} .{ }^{15}$ The depth of the hole was determined using fluoroscopy. Tapping and insertion of the screw was done without using the guide. For the pedicle screws in lumbar vertebrae in Cases 2 and 3, K-wires with a diameter of $3 \mathrm{~mm}$ were used to drill the hole under fluoroscopic control, confirming the depth of the K-wire penetration. After that, guidewires for the percutaneous pedicle screw system were inserted into the holes, and correctly sized pedicle screws were inserted after tapping along the guidewires.

\section{Case Series}

Case 1

A 68-year-old woman had undergone 5 anterior and posterior cervical spine surgeries for treating os odontoide$\mathrm{um}$ as a result of pseudarthrosis and infections. After solid fusion at $\mathrm{C1}-2$, she complained of gait disturbance with incomplete tetraplegia. MRI showed that the spinal cord was anteriorly compressed against the odontoid process because of severe deformity of the upper cervical region without stenosis of the vertebral canal. She was treated with occipitocervical fusion with bilateral C-3 and C-4 pedicle screws without cord decompression. Her symptoms diminished within 1 year after surgery. However, 6 years after surgery, she again complained of a slight gait disturbance. Lateral radiographs revealed pseudarthroses at the occiput- $\mathrm{C} 1$ with severe loosening of the pedicle screws (Fig. 5A). Because we thought that the instability between the occiput and C-1 caused her symptoms, we planned revision fusion surgery with posterior iliac bone grafting between the occiput and C-2. We thought that a strong anchor near the occipital bone should be selected to acquire rigid fixation that could promote bone fusion even in the case of restricted blood flow around the upper cervical area because of multiple surgeries. Because the C-3 and C-4 pedicles were severely damaged, we decided to use a right C-2 pedicle screw, which is thought to be a more reliable anchor than C-2 lamina screws or lateral mass screws into other subaxial vertebrae. ${ }^{5,10}$ Furthermore, we used lamina hooks and pedicle screws at C-7, T-1, and T-2 to add more rigidity. Before surgery, an FCTP model was designed using contrast-enhanced CT data as described above (Fig. 4)..$^{2}$ Because the screw trajectory into the right $\mathrm{C}-2$ pedicle was too narrow for a high-riding right vertebral artery (VA) ${ }^{9}$ and the insertion point of the screw was obscure and close to the right VA, we designed a CSI guide 



FIG. 3. Designing of the screw trajectory and the guide arm contact points. A: The screw trajectory is decided using a cylindrical segment, and the proper setting can be confirmed using sagittal, coronal, and axial (no data) views. B: To design contact areas of the guide, sphereshaped segments in diameters of $6-8 \mathrm{~mm}$ are located on the proper position. C: Next, bone tissue segments are subtracted from the sphere shaped segments, and the segmentation of the contact areas is finished. Figure is available in color online only.

for the surgery (Fig. 4). ${ }^{12,15}$ After confirming the conformation and stability of the guide (Fig. 5B), a pedicle screw was inserted without any complications. The patient's complaints diminished soon after surgery. Postoperative CT scans and radiographs showed accurate screw insertion, as in the preoperative plan (Fig. 5C). One year after the surgery, complete bone union between the occiput and C-2 was achieved (Fig. 5D), and at her most recent followup, 2 years after the last surgery, the patient had no trouble walking without a cane.

\section{Case 2}

A 74-year-old man receiving hemodialysis underwent posterior fusion surgery from L-2 to S-1 with instrumentation at another hospital. Eventually, all pedicle screws loosened completely, and all posterior implants had to be removed when the patient was sent to our hospital. He complained of severe back pain and showed severe sagittal imbalance (Fig. 6A and B). The L-4 and L-5 vertebrae

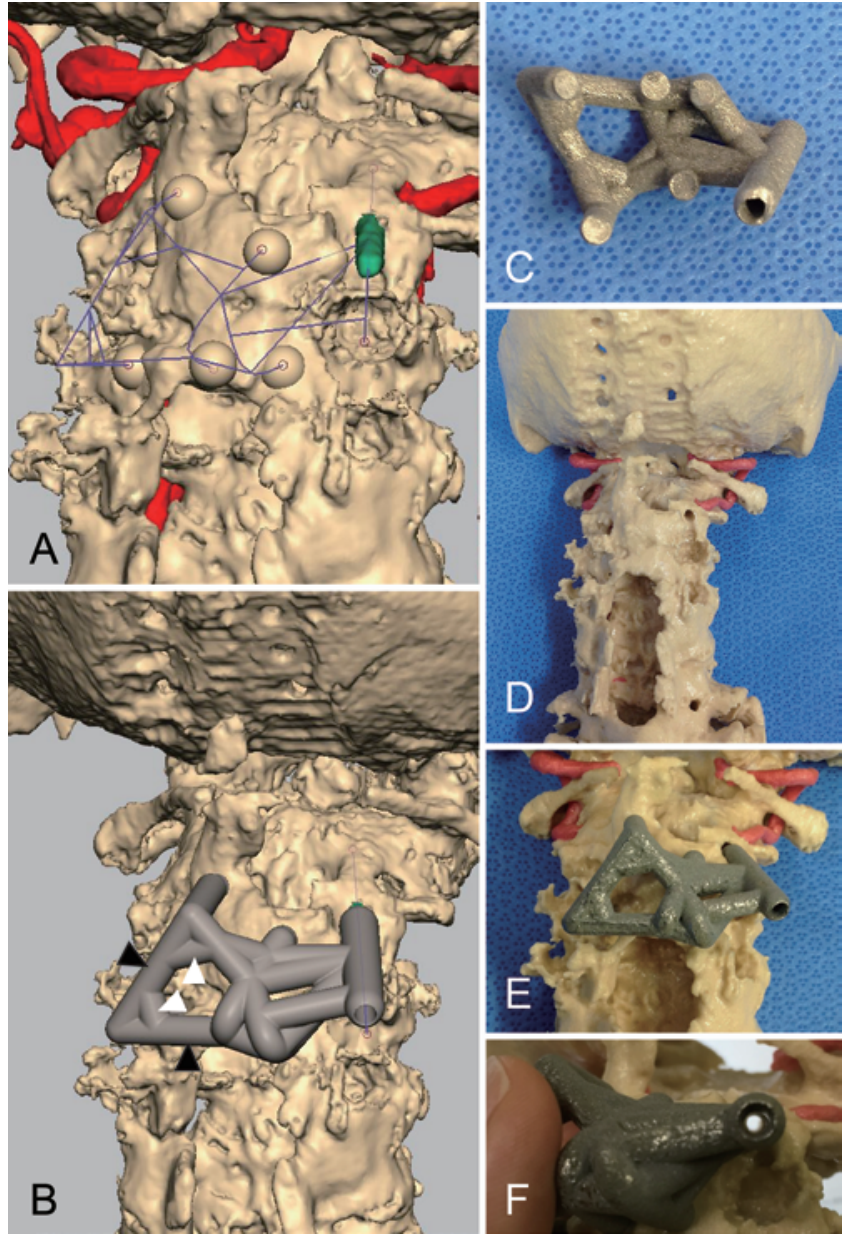

FIG. 4. Designing of the screw guide and the plaster model. A and B: The segmented areas are transferred to FreeForm software $(A)$ and the pedicle screw guide tubes are designed $(B)$. The guide tubes and contact points are connected with 5-mm-diameter curved cylindrical supports (B, black arrowheads) and reinforced by diagonal cylindrical supports (B, white arrowheads). C: Fabricated pure titanium screw guide. D: Full-scale plaster model with VAs. E: The fitting of the guide is confirmed on the plaster model. F: Because the plaster model has cylindrical holes corresponding to the pedicle screw trajectories, the accuracy of the fabrication and the fitting of the guide are checked as to whether the centers of the guide holes are on the same line as the centers of the cylindrical holes. Figure is available in color online only.

were fused, but the L2-3, L3-4, and L5-S1 levels showed pseudarthroses with destructive spondylolysis (Fig. 6B). We planned the revision fusion surgery from L-2 to the sacrum. Because the L-2 vertebra had 2 large bone defects along the previous pedicle screw trajectories (Fig. 6G), we planned to insert the pedicle screws with another trajectory from the tip of the superior articular facet of L-2 toward the anterior edge of the distal endplate (Fig. 6G), and an appropriate CSI guide was fabricated (Fig. 6C and D). During surgery, oblique lateral interbody fusion cages ${ }^{2}$ were inserted into the L2-3 and L3-4 levels from the right side, and an anterior cage was inserted at the L5-S1 level using an anterior retroperitoneal approach. After that, the laminae at L-2 to S-2 were exposed using a posterior approach. The CSI guide fitted well at the correct location and was fixed manually. Two pilot holes were made using 

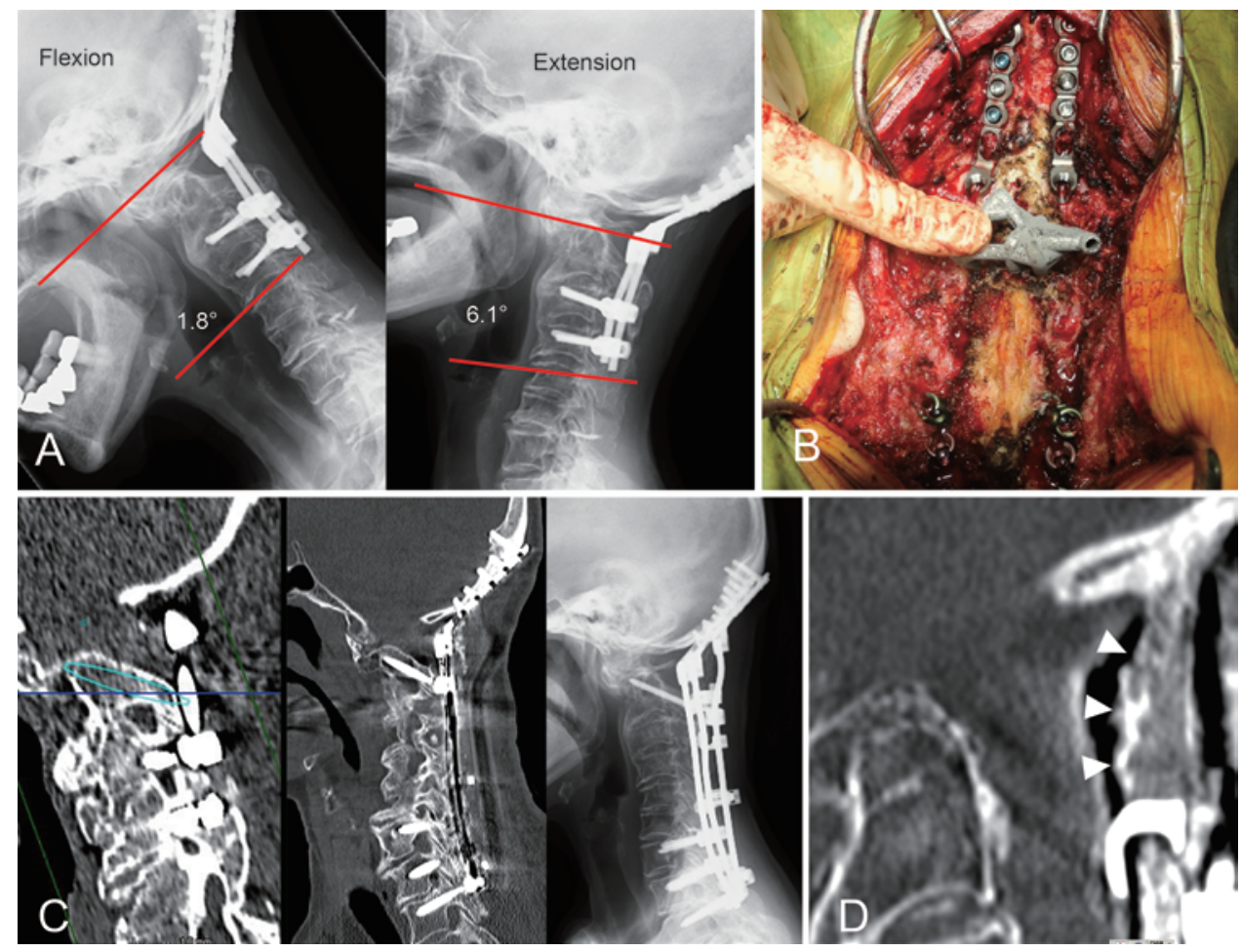

FIG. 5. Case 1. Images obtained in a 68-year-old female who underwent revision cervical spine fusion surgery. A: Lateral flexionextension radiograph showing pseudarthrosis between the occipital bone and C-1. The angles between the McGregor line and the base of the C-4 vertebra are $1.8^{\circ}$ and $6.1^{\circ}$ in flexion and extension positions, respectively. B: Placement of the titanium screw insertion guide during surgery. C: Planned screw trajectory, sagittal reconstructed CT scan, and lateral radiograph after the final surgery. D: A sagittal reconstructed CT view showing complete bone fusion between the occipital bone and C-2 lamina (white arrowheads). Figure is available in color online only.

3-mm K-wires, and the depth of the tip was confirmed using fluoroscopy (Fig. 6E and F). After this drilling, the CSI guide was removed and 2 guidewires were inserted into the same hole followed by tapping, and then 2 pedicle screws were set along the guidewires. We also inserted pedicle screws at other levels and bilateral S2-alar-iliac screws under fluoroscopy control, and these screws were connected with 6-mm titanium alloy rods. The patient's postoperative course was uneventful, and postoperative radiographs and CT scans showed accurate insertion of both screws into the L-2 pedicle (Fig. $6 \mathrm{G}$ and $\mathrm{H}$ ).

\section{Case 3}

A 64-year-old woman was referred to our hospital. The patient had undergone posterior spinal fusion surgery twice from the thorax to the sacrum at another hospital. She complained of severe back pain with radiating pain in her right leg. Lateral standing radiographs and CT images showed pseudarthroses at the L2-4 joints with bilateral rod breakage (Fig. 7A and B), and the right L-5 nerve root was compressed between the interbody cage and the right pedicle of L-5 (Fig. 7C). The posterior elements at L-4 and L-5 were completely fused with a previous posterior bone graft, and we anticipated that the insertion of pedicle screws to the L-4 vertebra would be difficult because of the absence of clear anatomical landmarks after multiple surgeries. Therefore, a CSI guide was designed and fabricated for the insertion of bilateral screws into the pedicle of L-4 (Fig. 7D, E, and H). After appropriate fitting to the plaster model was confirmed (Fig. 7E), the CSI guide was used in surgery. In this case, we first approached from the posterior side. All pedicle screws were exchanged with screws that were 1 or $2 \mathrm{~mm}$ larger in diameter than the original screws. After exposing the laminae at L-4 and L-5 and the right transverse process of L-4, the CSI guide was set correctly (Fig. 7F). The fitting of the guide was excellent, without any instability on the bone surface, and both pedicle screws were inserted (Fig. 7F and G). To correct the scoliosis between L-4 and L-5 and gain proper lordosis, posterior osteotomy was performed between the pedicles at L-4 and L-5 using a high-speed bur. Next, the lower part of the right pedicle of L-5 was removed in the same way to release the L-5 nerve root. Then, we performed lateral interbody fusion using a left lateral retroperitoneal approach. Two interbody cages at L2-3 and L3-4 were removed using the same approach, and oblique lateral interbody fusion cages were inserted into the L2-3, L3-4, and L4-5 levels. Finally, again using a posterior approach, all pedicle screws were connected with 6-mm titanium alloy rods. After the surgery, the patient's back pain and radiating pain in the right leg diminished within 2 weeks, and postoperative radiographs and CT scans showed accurate pedicle screw placement in both L-4 pedicles (Fig. 7H and I).

\section{Discussion}

A cervical pedicle screw ${ }^{1}$ is one of the most reliable 

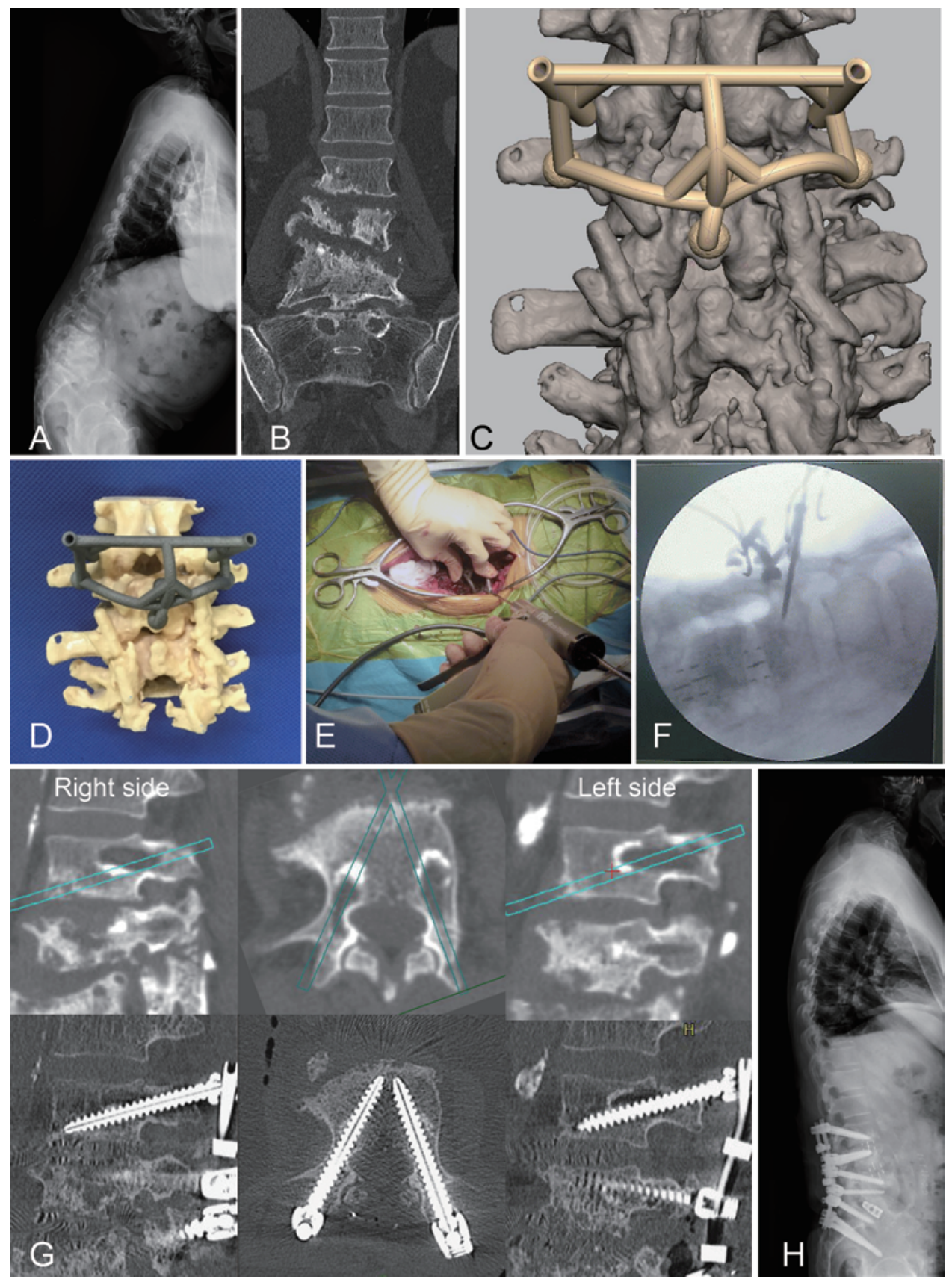

FIG. 6. Case 2. Images obtained in a 74-year-old male who underwent revision lumbar spine fusion surgery. A: Lateral standing radiograph obtained before revision surgery, showing severe sagittal imbalance. B: Coronal reconstructed CT scan obtained before revision surgery. C: Planning and designing of a bilateral L-2 pedicle screw insertion guide. D: Fabricated plaster model of the patient and an actual screw insertion guide. E: Two pilot holes were made using 3-mm K-wire. F: Fluoroscopic view obtained during the pilot hole drilling. G: Planning of the screw trajectories (upper pane/s) and corresponding reconstructed CT views after the revision surgery (lower panels). H: Lateral standing radiograph obtained after the revision surgery. Figure is available in color online only.

anchors used in several fixation techniques. ${ }^{5,10}$ However, its use can cause neurovascular injury. ${ }^{8}$ Pedicle screw insertion is particularly difficult during revision surgery because of morphological changes caused by laminectomy and/or bone grafting..$^{14}$ Navigation based on preoperative CT findings can be inaccurate because of artifacts from internal implants. One solution is intraoperative CT navigation, ${ }^{4}$ but the capital costs are high. Moreover, in revi- sion surgeries such as those described here, reliable reference frame fixation is often difficult because of severe iatrogenic bony destruction from previous techniques such as laminectomy. In such cases, the reference frame should be attached to a different vertebra or be a skull clamp, which can cause some positioning error during drilling or probing. Another solution is the use of image-based navigation templates. ${ }^{3,6,7,13}$ One of the advantages of such templates is 

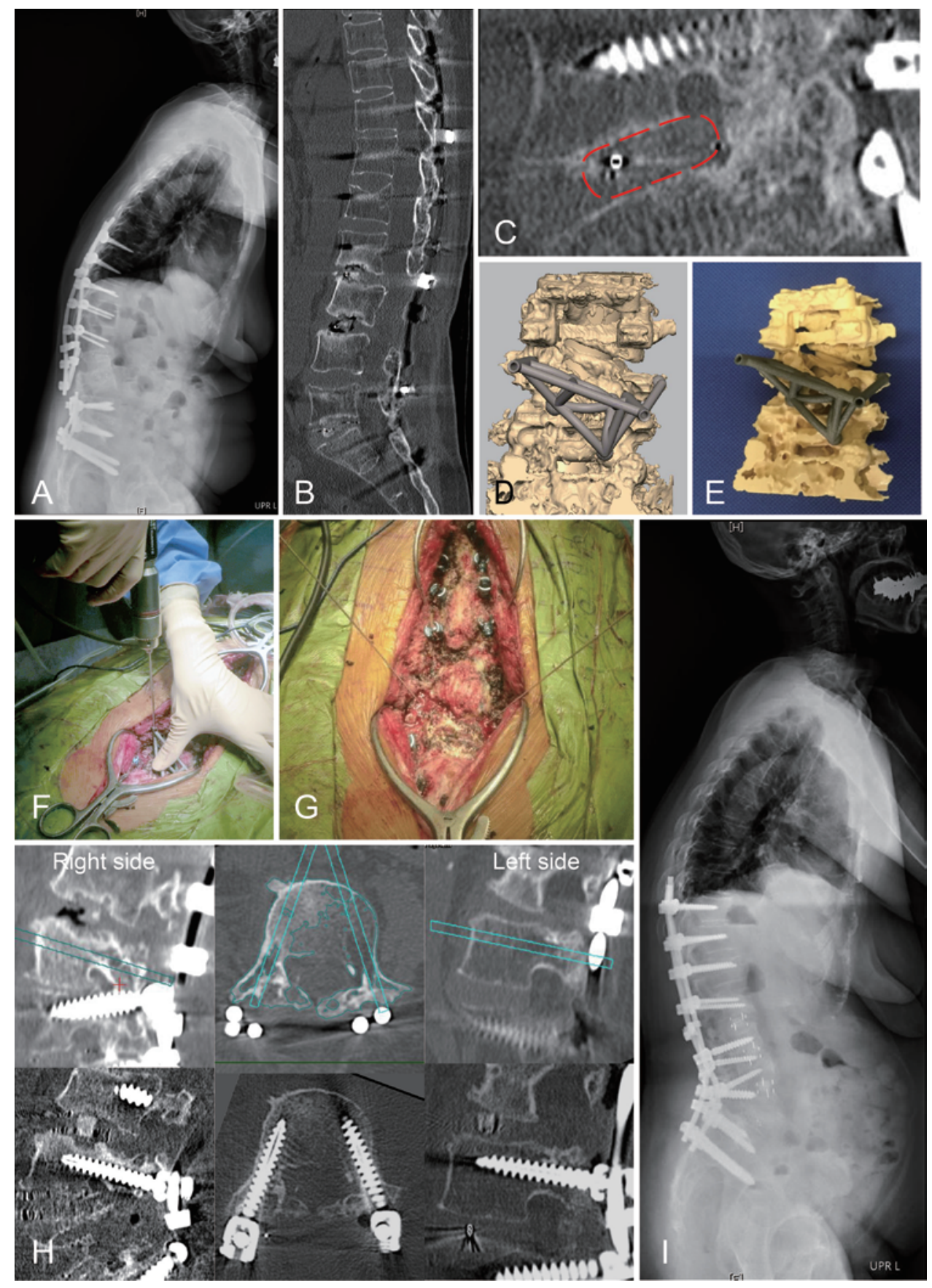

FIG. 7. Case 3. Images obtained in a 64-year-old female who underwent revision thoracolumbar spine fusion surgery. A: Lateral standing radiograph obtained before revision surgery, showing bilateral rod breakage and severe sagittal imbalance. B: Coronal reconstructed CT view obtained before revision surgery. C: The interbody cage on the right side compressed the right L-5 nerve root. The red dotted line shows the outline of the cage. D: Planning of a bilateral L-4 pedicle screw insertion guide. E: Fabricated plaster model of the patient and an actual screw insertion guide. F: Two pilot holes were made using a 3-mm K-wire. G: Two guidewires were inserted into the pilot hole. H: Planning of the screw trajectories (upper pane/s) and corresponding reconstructed CT views after the revision surgery (lower panels). I: A Lateral standing radiograph obtained after the revision surgery. Figure is available in color online only.

that the relative position between the template and the intended vertebra remains the same during the surgical procedure. However, these guides have not been used during revision surgery.

In this study, we used an FCTP model and a CSI guide for revision surgery of the cervical spine for the first time
(Case 1). The FCTP model proved useful for enhancing safe surgical exposure. Furthermore, the customized CSI guide could be fitted precisely to the bone surface during surgery, and the accuracy of screw positioning was established by postoperative CT scans. After the successful insertion of the pedicle screw and a satisfactory clinical 
result in Case 1, we used the same concept of CSI guides for 2 revision surgeries of the lumbar spine to confirm the guides' accuracy. In Case 2, both pedicles at L-2 had quite large bone defects because of loosening of the previous pedicle screws, and correct pedicle screw insertion with different trajectories was anticipated to be difficult if performed solely under fluoroscopic control. As expected, bilateral pedicle screws were inserted accurately into the L-2 vertebra using the CSI guide without any trouble (Fig. 6G and H). In Case 3, the insertion points of pedicle screws into the L-4 vertebra were not obvious because of a large posterior bone graft. We could easily fit the CSI guide onto the bone surface, and the pedicle screws were inserted with no trouble (Fig. 7F). In all 3 cases, it took a few minutes to fit the CSI guides to the bone surface, and we believe this method can save time compared with the image-based navigation technique. Because the followup periods of Cases 2 and 3 were less than 3 months, the long-term clinical efficacy of this approach needs to be confirmed.

The CSI guides had 5 or 6 small arms that could be fitted accurately to the bone surfaces (Fig. 4A-C). These contact areas were decided carefully based on the criteria listed above. Because we could not distinguish artifacts from bone tissue accurately, especially in osteoporotic bone regions, we selected areas with relatively high CT contrast values where the artifacts could be removed easily, which helped avoid any inaccuracy in fitting the guides. These guides are different from the conventional guides produced by simple virtual subtraction. The latter produce a wide contact area, whereas the relatively small footprint of our guide was advantageous when addressing cases with complex bone shapes or revision cases with artifacts (Fig. 1). The number of contact points and the target regions for such contact points are key factors in acquiring a precise fit between the guide and the bone surface. Surgeons should confirm the stability and the accuracy of the guide on the plaster model before surgery. Because the plaster model has cylindrical screw holes corresponding to the pedicle screw trajectories, the accuracy of the fabrication and the fitting of the guide are checked easily by manual inspection (Fig. 4F).

The accuracy of our novel guide in clinical use is one of its most significant problems. We have used this type of cutting guide with several small contact points for doing curved periacetabular osteotomy, and the accuracy of the guide was confirmed. ${ }^{12}$ Moreover, we have used the same concept of a screw insertion guide for primary spine surgery for 466 thoracic pedicle screws (36 patients with adolescent idiopathic scoliosis and 4 with ossification of the posterior longitudinal ligaments), and the success rate of screw placement was $98.7 \% .^{15}$ The concept of the CSI guide in revision surgery is essentially the same as in primary cases. The screw insertion was accurate for the 5 screws in this small study; however, because of artifacts and anatomical changes in revision surgeries, the contact points should be selected carefully according to the bone surface shape of each patient. Therefore, the success rate of screw placement in revision surgeries might be lower than in primary ones. The accuracy of the guide in revision surgery needs to be confirmed in a large study.
Maintaining the correct position of the guide during surgery is essential for accurate screw insertion. In these 3 cases, the CSI guides were pressed toward the bone surface by the surgeon's fingers. To keep the guides properly positioned during surgery, the shape of the guide should be ergonomically accurate. The inner diameter and the length of the guide tube also affect the accuracy of screw insertion. We set the guide tube to be $30 \mathrm{~mm}$ long with a 3.5$\mathrm{mm}$ inner diameter, the same as in our previous study. ${ }^{15}$ Theoretically, there is a little play between the guide and the probe or the K-wire, which will cause at most a 0.5 $\mathrm{mm}$ positional error from the ideal position at the tip of the screw if the screw is $30 \mathrm{~mm}$ long. Although the ideal size of the guide tube for clinical use should be decided in future studies, we believe our design of the guide tubes was safe enough for pedicle screw insertion even in the cervical spine.

One of the limitations of this technology is that it requires significant time, labor, and costs for preparation. ${ }^{15}$ It takes about 2-3 hours to design the CSI guide for each patient, 1 day for manufacturing, and 1 day for cleaning and sterilizing it. However, advances in 3D printing technology are expected to reduce the working time and cost in the future. Another limitation is that there is a possibility of deformation of the guide during fabrication and sterilization. The contact points of the guide are connected by curved cylindrical supports with a diameter of $5 \mathrm{~mm}$. If the supports are too long, they could yield a little during fabrication. To prevent such deformation, we added diagonal cylindrical supports (Fig. 4B, white arrowheads), and we confirmed the fitting of the guide to the plaster model after fabrication (Fig. 4E and F). As for sterilization, the CSI guides ware sterilized in an autoclave. Because they are made of pure titanium, we believe there is little chance of deformation of the guides during autoclaving, and this was indirectly clarified by the good clinical results in our previous report. ${ }^{15}$

\section{Conclusions}

In this study, we applied for the first time the novel CSI guides for revision spine surgeries, and all screws were inserted correctly without any complications. The CSI guides had several small contact areas, to fit the bone surface, which was different from the conventional guides with wide contact areas. Our novel CSI guides can be applied not only in primary spine-surgery cases, but also in revision surgeries with complex bone shapes or artifacts; however, the accuracy of our methods should be confirmed in a larger study in the future.

\section{Acknowledgments}

This study was supported by the program (Number 23-033) to develop medical equipment and devices to solve unmet medical needs from Japan's Ministry of Economy, Trade and Industry.

\section{References}

1. Abumi K, Kaneda K: Pedicle screw fixation for nontraumatic lesions of the cervical spine. Spine (Phila Pa 1976) 22:18531863,1997

2. Fujibayashi S, Hynes RA, Otsuki B, Kimura H, Takemoto M, 
Matsuda S: Effect of indirect neural decompression through oblique lateral interbody fusion for degenerative lumbar disease. Spine (Phila Pa 1976) 40:E175-E182, 2015

3. Hu Y, Yuan ZS, Spiker WR, Albert TJ, Dong WX, Xie H, et al: Deviation analysis of $\mathrm{C} 2$ translaminar screw placement assisted by a novel rapid prototyping drill template: a cadaveric study. Eur Spine J 22:2770-2776, 2013

4. Ishikawa Y, Kanemura T, Yoshida G, Matsumoto A, Ito Z, Tauchi R, et al: Intraoperative, full-rotation, three-dimensional image (O-arm)-based navigation system for cervical pedicle screw insertion. J Neurosurg Spine (Phila Pa 1976) 15:472-478, 2011

5. Jones EL, Heller JG, Silcox DH, Hutton WC: Cervical pedicle screws versus lateral mass screws. Anatomic feasibility and biomechanical comparison. Spine (Phila Pa 1976) 22:977-982, 1997

6. Kaneyama S, Sugawara T, Sumi M, Higashiyama N, Takabatake M, Mizoi K: A novel screw guiding method with a screw guide template system for posterior C-2 fixation: clinical article. J Neurosurg Spine 21:231-238, 2014

7. Lu S, Xu YQ, Lu WW, Ni GX, Li YB, Shi JH, et al: A novel patient-specific navigational template for cervical pedicle screw placement. Spine (Phila Pa 1976) 34:E959-E966, 2009

8. Neo M, Fujibayashi S, Miyata M, Takemoto M, Nakamura T: Vertebral artery injury during cervical spine surgery: a survey of more than 5600 operations. Spine (Phila Pa 1976) 33:779-785, 2008

9. Neo M, Matsushita M, Yasuda T, Sakamoto T, Nakamura $\mathrm{T}$ : Use of an aiming device in posterior atlantoaxial transarticular screw fixation. Technical note. J Neurosurg 97 (1 Suppl):123-127, 2002

10. Oda I, Abumi K, Sell LC, Haggerty CJ, Cunningham BW, McAfee PC: Biomechanical evaluation of five different occipito-atlanto-axial fixation techniques. Spine (Phila Pa 1976) 24:2377-2382, 1999

11. Otsuki B, Takemoto M, Fujibayashi S, Neo M, Kokubo T, Nakamura T: Pore throat size and connectivity determine bone and tissue ingrowth into porous implants: three-dimensional micro-CT based structural analyses of porous bioactive titanium implants. Biomaterials 27:5892-5900, 2006
12. Otsuki B, Takemoto M, Kawanabe K, Awa Y, Akiyama H, Fujibayashi S, et al: Developing a novel custom cutting guide for curved peri-acetabular osteotomy. Int Orthop 37:10331038,2013

13. Ryken TC, Owen BD, Christensen GE, Reinhardt JM: Imagebased drill templates for cervical pedicle screw placement. J Neurosurg Spine 10:21-26, 2009

14. Seichi A, Takeshita K, Nakajima S, Akune T, Kawaguchi H, Nakamura K: Revision cervical spine surgery using transarticular or pedicle screws under a computer-assisted imageguidance system. J Orthop Sci 10:385-390, 2005

15. Takemoto M, Fujibayashi S, Ota E, Otsuki B, Kimura H, Sakamoto T, et al: Additive-manufactured patient-specific titanium templates for thoracic pedicle screw placement: novel design with reduced contact area. Eur Spine J [epub ahead of print], 2015

\section{Disclosures}

The authors report no conflict of interest concerning the materials or methods used in this study or the findings specified in this paper.

\section{Author Contributions}

Conception and design: Otsuki, Takemoto. Acquisition of data: Otsuki, Masamoto. Analysis and interpretation of data: Otsuki, Fujibayashi. Drafting the article: Otsuki. Critically revising the article: Takemoto, Fujibayashi, Kimura, Masamoto, Matsuda. Reviewed submitted version of manuscript: Otsuki, Takemoto, Fujibayashi, Kimura, Matsuda. Approved the final version of the manuscript on behalf of all authors: Otsuki. Study supervision: Matsuda.

\section{Correspondence}

Bungo Otsuki, Department of Orthopaedic Surgery, Graduate School of Medicine, Kyoto University, 54 Kawahara-cho, Shogoin, Sakyo-ku, Kyoto City, Kyoto 606-8507, Japan. email: bungo@kuhp.kyoto-u.ac.jp. 